\title{
ON THE REAL RANK OF $C^{*}$-ALGEBRAS OF NILPOTENT LOCALLY COMPACT GROUPS
}

\author{
ROBERT J. ARCHBOLD and EBERHARD KANIUTH*
}

\begin{abstract}
If $G$ is an almost connected, nilpotent, locally compact group then the real rank of the $C^{*}$-algebra $C^{*}(G)$ is given by $\operatorname{RR}\left(C^{*}(G)\right)=\operatorname{rank}(G /[G, G])=\operatorname{rank}\left(G_{0} /\left[G_{0}, G_{0}\right]\right)$, where $G_{0}$ is the connected component of the identity element. In particular, for the continuous Heisenberg group $G_{3}, \operatorname{RR}\left(C^{*}\left(G_{3}\right)\right)=2$.
\end{abstract}

\section{Introduction}

For a $C^{*}$-algebra $A$, the real rank $\operatorname{RR}(A)[5]$ and the stable rank $\operatorname{sr}(A)[21]$ have been defined as non-commutative analogues of the real and complex dimension of topological spaces. Several authors have computed or estimated the real and stable rank of group $C^{*}$-algebras $C^{*}(G)$ for various classes of locally compact groups $G$ [2], [3], [10], [11], [14], [18], [22], [23], [24], [25], [26], [27], [28], [29]. In generalizing the result of Sudo and Takai [28] for simply connected nilpotent Lie groups, it was shown in [3] that the stable rank of the $C^{*}$-algebra of an almost connected, nilpotent group $G$ is given by the formula

$$
\operatorname{sr}\left(C^{*}(G)\right)=1+\left\lfloor\frac{1}{2} \operatorname{rank}(G /[G, G])\right\rfloor
$$

and hence that the real rank $\operatorname{RR}\left(C^{*}(G)\right)$ satisfies

$$
\operatorname{rank}(G /[G, G]) \leq \operatorname{RR}\left(C^{*}(G)\right) \leq \operatorname{rank}(G /[G, G])+1,
$$

with equality on the left if the $\operatorname{rank}$ of $G /[G, G]$ is odd.

Subsequently, L. G. Brown [4] has made an incisive analysis of the behaviour of real and stable rank in CCR (liminal) $C^{*}$-algebras $A$, partly based on the notion of the topological dimension top $\operatorname{dim}(A)$ which was introduced in his earlier work with Pedersen [6]. By using these results, we are now able to

${ }^{*}$ We are grateful to Larry Brown for helpful discussions concerning [4]. We are also grateful to the referee for helpful comments and for suggesting a simplification in part of the proof of Proposition 2.4.

Received 17 August 2010. 
show that $\operatorname{RR}\left(C^{*}(G)\right)=\operatorname{rank}(G /[G, G])$ in all cases. Furthermore, Brown's results allow us to give simultaneously a similar approach to equation (1.1). This enables us to close a gap in [3, Theorem 1.5] arising from the use of [19, Lemma 2] which omits the statement of an implicit dimensional condition. The significance of dimension is illustrated by the recent example due to Kucerovsky and $\mathrm{Ng}$ [15] of a non-stable, continuous trace $C^{*}$-algebra $A$, all of whose irreducible representations are infinite dimensional, such that $\operatorname{dim} \hat{A}=\operatorname{sr}(A)=\infty$. Accordingly, in the next section we shall estimate top $\operatorname{dim}\left(C^{*}(G)\right)$ for certain groups $G$.

The main result on real rank is obtained in Theorem 3.4. As a corollary, we settle a dichotomy for the real rank of the $C^{*}$-algebra of the continuous Heisenberg group: the value is 2 rather than 3 .

We conclude this section with some definitions and notation. For a discrete, torsion-free, abelian group $D$, rank $D$ means the torsion-free rank of $D$ (see [13]). That is, rank $D$ is the maximal number of independent elements if this is finite and rank $D=\infty$ otherwise. For a general locally compact group $G$, let $G^{c}$ denote the set of all compact elements of $G$, where an element is called compact if it generates a relatively compact subgroup. If $G$ is a locally compact group with relatively compact conjugacy classes, then $G^{c}$ is a closed normal subgroup of $G$ and $G / G^{c}$ is a compact-free, locally compact, abelian group [12, Theorem 3.16]. As such, $G / G^{c}$ has the form $R^{k} \times D$ where $D$ is a torsion-free discrete group. The rank of $G$ is then defined to be $k+\operatorname{rank} D$. In particular, rank $D<\infty$ whenever $G / G^{c}$ is compactly generated.

Finally, for any locally compact group $G$, we will denote by $G_{0}$ the connected component of the identity element, by $[G, G]$ the closed commutator subgroup of $G$ and by $G_{F}$ the subgroup of $G$ consisting of all elements with relatively compact conjugacy classes. The group $G$ is said to be almost connected if the quotient group $G / G_{0}$ is compact.

\section{The topological dimension of some group $C^{*}$-algebras}

Let $A$ be a type $I C^{*}$-algebra. It is well-known that the spectrum $\hat{A}$ is homeomorphic to the primitive ideal space $\operatorname{Prim}(A)$, via the map which sends the unitary equivalence class $[\pi]$ of an irreducible representation $\pi$ to the primitive ideal ker $\pi$, and that both spaces are almost Hausdorff in the sense that every non-empty closed subset contains a non-empty, relatively open, Hausdorff subset. It follows from [6, 2.2(v) and Remark 2.5(ii)] that the topological dimension of $A$ is given by

top $\operatorname{dim}(A)=\sup \{\operatorname{dim} K: K$ a compact Hausdorff subset of $\hat{A}\} \in[0, \infty]$, where $\operatorname{dim} K$ is the covering dimension of $K$ (see [20]). 
The next lemma is essentially a special case of [6, Proposition 2.3], but it is convenient to express it in the following form.

Lemma 2.1. Let $A$ be a type I $C^{*}$-algebra and let $\emptyset=V_{0} \subseteq V_{1} \subseteq V_{2} \subseteq$ $\cdots \subseteq V_{n}=\hat{A}$ be an increasing finite sequence of open subsets of $\hat{A}$. Then

top $\operatorname{dim}(A)=\max _{1 \leq j \leq n} \sup \left\{\operatorname{dim} K: K\right.$ a compact Hausdorff subset of $\left.V_{j} \backslash V_{j-1}\right\}$.

Proof. For $1 \leq j \leq n$, let $I_{j}$ be the closed two-sided ideal of $A$ such that $\hat{I}_{j}=V_{j}$ (so that $I_{n}=A$ ). Then $I_{j} \widehat{/ I_{j-1}}=V_{j} \backslash V_{j-1}$ and so by [6, Proposition 2.3]

top $\operatorname{dim}(A)$

$$
\begin{aligned}
& =\max _{1 \leq j \leq n} \operatorname{top} \operatorname{dim}\left(I_{j} / I_{j-1}\right) \\
& =\max _{1 \leq j \leq n} \sup \left\{\operatorname{dim} K: K \text { a compact Hausdorff subset of } V_{j} \backslash V_{j-1}\right\} .
\end{aligned}
$$

Proposition 2.2. Let $G$ be a connected, simply connected, nilpotent Lie group of dimension $d$. Then top $\operatorname{dim}\left(C^{*}(G)\right) \leq d$.

Proof. Let $\mathrm{g}$ be the Lie algebra associated with $G$ and let $k: \mathfrak{g}^{*} \rightarrow \hat{G}$ be the Kirillov map, which is an open, continuous surjection. By [7, Theorem 3.1.14], there is a strictly increasing sequence $\emptyset=U_{0} \subset U_{1} \subset U_{2} \subset \cdots \subset U_{n}=\mathrm{g}^{*}$ of $\operatorname{Ad}^{*}(G)$-invariant open subsets of $\mathrm{g}^{*}$ and, for $1 \leq j \leq n$, a vector subspace $W_{j}$ of $\mathrm{g}^{*}$ of (linear) dimension $m_{j}$ and a homeomorphism

$$
\psi_{j}: U_{j} \backslash U_{j-1} \rightarrow\left(\left(U_{j} \backslash U_{j-1}\right) \cap W_{j}\right) \times \mathrm{R}^{d-m_{j}}
$$

such that

(i) the restriction $k_{j}$ of $k$ to $\left(U_{j} \backslash U_{j-1}\right) \cap W_{j}$ is a (continuous) bijection onto $k\left(U_{j}\right) \backslash k\left(U_{j-1}\right)$,

(ii) $\left.k\right|_{U_{j} \backslash U_{j-1}}=k_{j} \circ P_{j} \circ \psi_{j}$, where $P_{j}$ is the projection from $\left(\left(U_{j} \backslash U_{j-1}\right) \cap\right.$ $\left.W_{j}\right) \times \mathrm{R}^{d-m_{j}}$ onto $\left(U_{j} \backslash U_{j-1}\right) \cap W_{j}$.

Let $V_{j}=k\left(U_{j}\right)$, an open subset of $\hat{G}(1 \leq j \leq n)$. Note that $V_{j} \backslash V_{j-1}=$ $k\left(U_{j} \backslash U_{j-1}\right)$ since $U_{j-1}$ is $\operatorname{Ad}^{*}(G)$-invariant.

Temporarily fix $j$ and let $E$ be an open subset of $\left(U_{j} \backslash U_{j-1}\right) \cap W_{j}$. Then $\psi_{j}^{-1}\left(E \times \mathrm{R}^{d-m_{j}}\right)=F_{j} \cap\left(U_{j} \backslash U_{j-1}\right)$ for some open subset $F_{j}$ of $U_{j}$. Hence, by (ii) and the fact that $U_{j}$ and $U_{j-1}$ are $\operatorname{Ad}^{*}(G)$-invariant,

$$
k_{j}(E)=k\left(F_{j} \cap\left(U_{j} \backslash U_{j-1}\right)\right)=k\left(F_{j}\right) \cap\left(V_{j} \backslash V_{j-1}\right),
$$


which is open in $V_{j} \backslash V_{j-1}$ since $F_{j}$ is open in $\mathrm{g}^{*}$. Thus $k_{j}$ is a homeomorphism (and so its image $V_{j} \backslash V_{j-1}$ is, in fact, Hausdorff). Let $K$ be a compact, Hausdorff subset of $V_{j} \backslash V_{j-1}$. Then $k_{j}^{-1}(K)$ is a compact subset of $\left(U_{j} \backslash U_{j-1}\right) \cap W_{j}$ and hence is a closed subset of the Hausdorff space $W_{j}$. By [20, Chapter 3, Proposition 1.5] and the fact that the linear and covering dimensions of $W_{j}$ coincide, we have $\operatorname{dim} K \leq \operatorname{dim}\left(W_{j}\right)=m_{j}$. Since $m_{j} \leq d$ and $j$ was arbitrary, top $\operatorname{dim}\left(C^{*}(G)\right) \leq d$ by Lemma 2.1 .

In the context of Proposition 2.2, if $G$ is abelian then

$$
\text { top } \operatorname{dim}\left(C^{*}(G)\right)=\text { top } \operatorname{dim}\left(C_{0}\left(\mathrm{R}^{d}\right)\right)=d .
$$

If $G$ is non-abelian then $m_{j} \leq d-2(1 \leq j \leq n-1)$ and, although $m_{n}=d$, if $K$ is a compact Hausdorff subset of $\hat{G} \backslash V_{n-1}$ then

$$
\begin{aligned}
\operatorname{dim} K=\operatorname{dim}\left(k_{n}^{-1}(K)\right) & \leq \operatorname{dim}\left(\mathfrak{g}^{*} \backslash U_{n-1}\right)=\operatorname{dim}(G /[G, G])^{\wedge} \\
& =\operatorname{rank}(G /[G, G]) \leq d-1 .
\end{aligned}
$$

Thus top $\operatorname{dim}\left(C^{*}(G)\right) \leq d-1$, with equality in the case of the Heisenberg group $(d=3)$.

Corollary 2.3. Let $G$ be a connected, nilpotent Lie group. Then top $\operatorname{dim}\left(C^{*}(G)\right)<\infty$.

Proof. Let $H$ be the simply connected covering group of $G$. Then $C^{*}(G)$ is a quotient of $C^{*}(H)$ and, in particular, any compact Hausdorff subset of $\hat{G}$ is homeomorphic to a compact Hausdorff subset of $\hat{H}$ (see also [6, Proposition 2.4]). Hence

$$
\text { top } \operatorname{dim}\left(C^{*}(G)\right) \leq \text { top } \operatorname{dim}\left(C^{*}(H)\right)<\infty,
$$

by Proposition 2.2.

We recall from $[8,4.7 .12]$ that a generalised continuous trace $C^{*}$-algebra $A$ of finite length has a composition series

$$
\{0\}=I_{0} \subseteq I_{1} \subseteq I_{2} \subseteq \cdots \subseteq I_{n}=A
$$

such that $I_{j} / I_{j-1}=J\left(A / I_{j-1}\right)$ for $1 \leq j \leq n$ (where the ideal $J(B)$ of a $C^{*}$-algebra $B$ is the closed linear span of the set of positive elements with finite, continuous trace on the spectrum $\hat{B}$ ). For $1 \leq j \leq n$, write $V_{j}=\hat{I}_{j}$, an open subset of $\hat{A}$. Since $I_{j} / I_{j-1}$ is a continuous trace $C^{*}$-algebra, $V_{j} \backslash V_{j-1}$ is a locally compact Hausdorff space for $1 \leq j \leq n$ (where $V_{0}=\emptyset$ ). Now suppose that $G$ is a group of automorphisms of $A$. Since $I_{1}=J(A), I_{1}$ is $G$ invariant and so $V_{1}$ is invariant for the action of $G$ on $\hat{A}$. Since $I_{1}$ is $G$-invariant, 
there is an induced action of $G$ on $A / I_{1}$. Since $I_{2} / I_{1}=J\left(A / I_{1}\right), I_{2} / I_{1}$ is $G$ invariant. It follows that $I_{2}$ is $G$-invariant and that $V_{2}$ is a $G$-invariant subset of $\hat{A}$. Proceeding by induction, we obtain that $I_{j}$ and $V_{j}$ are $G$-invariant for $1 \leq j \leq n$.

Proposition 2.4. Let $G$ be a locally compact group containing a closed, normal, second countable subgroup $N$ of finite index such that $C^{*}(N)$ is a generalised continuous trace $C^{*}$-algebra of finite length. Then

$$
\text { top } \operatorname{dim}\left(C^{*}(G)\right) \leq \text { top } \operatorname{dim}\left(C^{*}(N)\right) \text {. }
$$

Proof. The group $G$ acts on $N$ via the restriction of inner automorphisms. The induced action of $G$ on $\hat{N}$ is given by $g \cdot \tau(n)=\tau\left(g^{-1} n g\right)(g \in G, \tau \in$ $\hat{N}, n \in N)$ and hence $g \cdot \tau$ is unitarily equivalent to $\tau$ whenever $g \in N$.

Since $C^{*}(N)$ is CCR $[8,4.7 .12(\mathrm{c})]$ and $G / N$ is finite, the $G$-orbits

$$
G(\tau):=\{g \cdot \tau: g \in G\} \quad(\tau \in \hat{N})
$$

are finite closed sets. Let $\hat{N} / G$ be the quotient space, consisting of all $G$ orbits. Then the quotient map $q: \hat{N} \rightarrow \hat{N} / G$ is continuous and also open since $\left.q^{-1}(q(V))\right)=\bigcup_{g \in G} g \cdot V$ is open in $\hat{N}$ for every open subset $V$ of $\hat{N}$. If $\pi \in \hat{G}$ then $\operatorname{supp}\left(\left.\pi\right|_{N}\right)=G(\tau)$ for some $\tau \in \hat{N}$. Conversely, if $\tau \in \hat{N}$ and $\pi$ is any element of $\operatorname{supp}\left(\operatorname{ind}_{N}^{G} \tau\right)$, then $\operatorname{supp}\left(\left.\pi\right|_{N}\right)=G(\tau)$. Thus we have a surjective mapping $r: \hat{G} \rightarrow \hat{N} / G$ defined by $r(\pi)=\operatorname{supp}\left(\left.\pi\right|_{N}\right)$. It follows from the continuity of restricting representations that $r$ is continuous.

Let $\sigma \in \widehat{N} / G$ and choose $\tau \in \widehat{N}$ such that $G(\tau)=\sigma$. We claim that $r^{-1}(\sigma)$ is finite. Note first that $r^{-1}(\sigma)=\operatorname{supp}\left(\operatorname{ind}_{N}^{G} \tau\right)$. It therefore suffices to show that the commutant of $\operatorname{ind}_{N}^{G} \tau$ is finite dimensional. This can be deduced from the work of Mackey and Blattner on induced representations. For the reader's convenience, we indicate the argument. To fix notation, given two representations $\pi$ and $\rho$ of $G$, let $\operatorname{Hom}_{G}(\pi, \rho)$ denote the space of bounded linear operators from $H(\pi)$ into $H(\rho)$ (the Hilbert spaces of $\pi$ and $\rho$, respectively) intertwining $\pi$ and $\rho$.

Now let $\pi=\operatorname{ind}_{N}^{G} \tau$, where $\tau$ is as above, and realize $H(\pi)$ as the space of all continuous mappings $\xi: G \rightarrow H(\tau)$ satisfying the covariance condition $\xi(x n)=\tau\left(n^{-1}\right) \xi(x)$ for all $x \in G$ and $n \in N$. Then $\pi(x)$ acts on $\xi$ by $\pi(x) \xi(y)=\xi\left(x^{-1} y\right), y \in G$. Fix a finite set $X$ of representatives for the cosets of $N$ in $G$, and to any $T \in \operatorname{Hom}_{N}\left(\tau,\left.\rho\right|_{N}\right)$ associate an operator $\phi(T)$ : $H(\pi) \rightarrow H(\rho)$ by setting

$$
\phi(T) \xi=\sum_{x \in X} \rho(x) T(\xi(x)), \quad \xi \in H(\pi) .
$$


It is then straightforward to verify that this definition does not depend on the choice of $X$ and that the map $T \rightarrow \phi(T)$ is a linear isomorphism from $\operatorname{Hom}_{N}\left(\tau,\left.\rho\right|_{N}\right)$ onto $\operatorname{Hom}_{G}(\pi, \rho)$. Now take $\rho=\pi$ and observe that $\operatorname{Hom}_{N}\left(\tau,\left.\pi\right|_{N}\right)$ is finite dimensional since $\operatorname{supp}\left(\left.\pi\right|_{N}\right)=G(\tau)$ is finite. It follows that $\operatorname{Hom}_{G}\left(\operatorname{ind}_{N}^{G} \tau, \operatorname{ind}_{N}^{G} \tau\right)$ is finite dimensional.

Since $C^{*}(N)$ is a generalised continuous trace $C^{*}$-algebra of finite length, there is a sequence $\emptyset=V_{0} \subseteq V_{1} \subseteq V_{2} \subseteq \cdots \subseteq V_{n}=\hat{N}$ of open subsets of $\hat{N}$, which are invariant for the action of $G$ on $\hat{N}$, such that $V_{j} \backslash V_{j-1}$ is a locally compact Hausdorff space for $1 \leq j \leq n$. Suppose that $\tau_{1}, \tau_{2} \in V_{j} \backslash V_{j-1}$ are such that $q\left(\tau_{1}\right) \neq q\left(\tau_{2}\right)$. Then the disjoint finite subsets $G\left(\tau_{1}\right)$ and $G\left(\tau_{2}\right)$ can be separated by $G$-invariant open subsets of $V_{j} \backslash V_{j-1}$. Since the restriction of $q$ from $V_{j} \backslash V_{j-1}$ onto $q\left(V_{j} \backslash V_{j-1}\right)=q\left(V_{j}\right) \backslash q\left(V_{j-1}\right)$ is open, $q\left(\tau_{1}\right)$ and $q\left(\tau_{2}\right)$ can be separated by open subsets of $q\left(V_{j} \backslash V_{j-1}\right)$. So $q\left(V_{j} \backslash V_{j-1}\right)$ is a locally compact Hausdorff space.

We define a sequence $\emptyset=U_{0} \subseteq U_{1} \subseteq \cdots \subseteq U_{n}=\hat{G}$ of open subsets of $\hat{G}$ by $U_{j}=r^{-1}\left(q\left(V_{j}\right)\right)(0 \leq j \leq n)$. Let $K$ be any compact Hausdorff subset of $U_{j} \backslash U_{j-1}$. Then $r(K)$ is a compact subset of the Hausdorff space $q\left(V_{j} \backslash V_{j-1}\right)$. Since $N$ is second countable, $C^{*}(N)$ is separable and so $\hat{N}$ is second countable. Hence $\hat{N} / G$ and $r(K)$ are second countable and so the compact Hausdorff space $r(K)$ is metrizable. Since $r^{-1}(\sigma)$ is finite for each $\sigma \in \hat{N} / G$ and $\left.r\right|_{K}: K \rightarrow r(K)$ is a continuous closed surjection, it follows from [20, Chapter 9, Proposition 2.6] that $\operatorname{dim} r(K) \geq \operatorname{dim} K$. On the other hand, since the action of $G$ on $\hat{N}$ factors through the finite group $G / N, C:=$ $q^{-1}(r(K))$ is a compact subset of the Hausdorff space $V_{j} \backslash V_{j-1}$ and $q^{-1}(\sigma)$ is finite for each $\sigma \in \hat{N} / G$. Since $C$ is $G$-invariant, the restriction of the open mapping $q$ to $C$ is also open and so it follows from [20, Chapter 9, Proposition 2.16] that $\operatorname{dim} C=\operatorname{dim} r(K)$. Thus $\operatorname{dim} K \leq \operatorname{dim} C$. It now follows from Lemma 2.1 that top $\operatorname{dim}\left(C^{*}(G)\right) \leq \operatorname{top} \operatorname{dim}\left(C^{*}(N)\right)$.

THEOREM 2.5. Let $G$ be a locally compact group containing a closed normal subgroup $N$ of finite index such that $N$ is a connected, nilpotent Lie group. Then

$$
\text { top } \operatorname{dim}\left(C^{*}(G)\right) \leq \text { top } \operatorname{dim}\left(C^{*}(N)\right)<\infty .
$$

Proof. Since $N$ is a connected Lie group, it is second countable. Furthermore, since $N$ is also nilpotent, $C^{*}(N)$ is a generalised continuous trace $C^{*}$-algebra of finite length by [9]. So by Proposition 2.4 and Corollary 2.3, top $\operatorname{dim}\left(C^{*}(G)\right) \leq$ top $\operatorname{dim}\left(C^{*}(N)\right)<\infty$.

If $G$ is an almost connected, locally compact group with $G_{0}$ nilpotent, then the pro-Lie structure utilised in the proof of Lemma 3.2 below can be com- 
bined with the result of Theorem 2.5 to yield the fact that top $\operatorname{dim}\left(C^{*}(G)\right) \leq$ top $\operatorname{dim}\left(C^{*}\left(G_{0}\right)\right)$.

\section{Almost connected, nilpotent groups}

In the first two results of this section, it suffices to assume that $G_{0}$ is nilpotent (rather than $G$ itself).

Lemma 3.1. Let $G$ be a locally compact group such that $G_{0}$ is a nilpotent Lie group and $G / G_{0}$ is finite. Then

(1) $\operatorname{RR}\left(C^{*}(G)\right) \leq \max \left\{1, \operatorname{rank}\left(G_{0} /\left[G_{0}, G_{0}\right]\right)\right\}$,

(2) $\operatorname{sr}\left(C^{*}(G)\right) \leq \max \left\{2,1+\left\lfloor\frac{1}{2} \operatorname{rank}\left(G_{0} /\left[G_{0}, G_{0}\right]\right)\right\rfloor\right\}$.

Proof. Let $A=C^{*}(G)$, a CCR $C^{*}$-algebra. As in the proof of [3, Lemma 1.2], $A$ has a closed two-sided ideal $I$, all of whose irreducible representations are infinite dimensional, such that $A / I$ is isomorphic to $C^{*}\left(G /\left[G_{0}, G_{0}\right]\right)$, all of whose irreducible representations are finite dimensional. Since $A$ is CCR, it follows from [4, Theorem 3.6] that $\mathrm{RR}(A)=\max \{\mathrm{RR}(I), \mathrm{RR}(A / I)\}$ and similarly for the stable rank. Furthermore, by [6, Proposition 2.2] and Theorem 2.5, top $\operatorname{dim}(I) \leq \operatorname{top} \operatorname{dim}(A)<\infty$. Since all of the irreducible representations of $I$ are infinite dimensional, it follows from [4, Theorem 3.10] that $\mathrm{RR}(I) \leq 1$ and $\operatorname{sr}(I) \leq 2$. Thus

$$
\operatorname{RR}\left(C^{*}(G)\right) \leq \max \left\{1, \operatorname{RR}\left(C^{*}\left(G /\left[G_{0}, G_{0}\right]\right)\right)\right\}
$$

and

$$
\operatorname{sr}\left(C^{*}(G)\right) \leq \max \left\{2, \operatorname{sr}\left(C^{*}\left(G /\left[G_{0}, G_{0}\right]\right)\right)\right\} .
$$

We temporarily write $N=\left[G_{0}, G_{0}\right]$. Since $\left[G / N: G_{0} / N\right]=\left[G: G_{0}\right]<$ $\infty$ and $G_{0} / N$ is abelian, $G_{0} / N$ is contained in $(G / N)_{F}$ with necessarily finite index (where $(G / N)_{F}$ is the subgroup of $G / N$ consisting of all elements with relatively compact conjugacy classes). It follows from [2, Lemma 2.8] that $\operatorname{rank}\left((G / N)_{F}\right)=\operatorname{rank}\left(G_{0} / N\right)$. Since $G / N$ is a Moore group [17], it follows from [2, Theorem 3.4] that

$$
\operatorname{RR}\left(C^{*}(G / N)\right) \leq \operatorname{rank}\left((G / N)_{F}\right)=\operatorname{rank}\left(G_{0} / N\right)
$$

and also

$$
\operatorname{sr}\left(C^{*}(G / N)\right) \leq 1+\left\lfloor\frac{1}{2} \operatorname{rank}\left(G_{0} / N\right)\right\rfloor,
$$

as required. (This estimate for $\operatorname{sr}\left(C^{*}(G / N)\right)$ can also be obtained from [22, Corollary 3.3].) 
LEMMA 3.2. Let $G$ be an almost connected, locally compact group such that $G_{0}$ is nilpotent. Then

(1) $\operatorname{RR}\left(C^{*}(G)\right) \leq \max \left\{1, \operatorname{rank}\left(G_{0} /\left[G_{0}, G_{0}\right]\right)\right\}$,

(2) $\operatorname{sr}\left(C^{*}(G)\right) \leq \max \left\{2,1+\left\lfloor\frac{1}{2} \operatorname{rank}\left(G_{0} /\left[G_{0}, G_{0}\right]\right)\right\rfloor\right\}$.

Proof. By [16, Theorem 4.6], $G$ is a projective limit of Lie groups $G / K_{\alpha}$, where the $K_{\alpha}$ are compact normal subgroups of $G$, and by [3, Lemma 1.1] $\operatorname{RR}\left(C^{*}(G)\right)=\sup _{\alpha} \operatorname{RR}\left(C^{*}\left(G / K_{\alpha}\right)\right)$ and similarly for the stable rank. So let $K$ be any compact normal subgroup of $G$ such that $G / K$ is a Lie group. It suffices to show that

$$
\operatorname{RR}\left(C^{*}(G / K)\right) \leq \max \left\{1, \operatorname{rank}\left(G_{0} /\left[G_{0}, G_{0}\right]\right)\right\}
$$

and that $\operatorname{sr}\left(C^{*}(G / K)\right) \leq \max \left\{2,1+\left\lfloor\frac{1}{2} \operatorname{rank}\left(G_{0} /\left[G_{0}, G_{0}\right]\right)\right\rfloor\right\}$.

For this, we use some facts from [3, p. 94]. Firstly, $(G / K)_{0}=\left(G_{0} K\right) / K$ and this is nilpotent since it is a quotient of $G_{0}$. Then $(G / K) /(G / K)_{0}=$ $G / G_{0} K$ and this is finite since it is both discrete and also a quotient of the compact group $G / G_{0}$. Finally,

$$
\operatorname{rank}\left((G / K)_{0} /\left[(G / K)_{0},(G / K)_{0}\right]\right) \leq \operatorname{rank}\left(G_{0} /\left[G_{0}, G_{0}\right]\right) .
$$

It follows from Lemma 3.1 that

$$
\begin{aligned}
\mathrm{RR}\left(C^{*}(G / K)\right) & \leq \max \left\{1, \operatorname{rank}\left((G / K)_{0} /\left[(G / K)_{0},(G / K)_{0}\right]\right)\right\} \\
& \leq \max \left\{1, \operatorname{rank}\left(G_{0} /\left[G_{0}, G_{0}\right]\right)\right\}
\end{aligned}
$$

and a similar argument applies to the stable rank.

The following corollary is an extension of [3, Proposition 2.6].

COROLlARY 3.3. Let $G$ be a locally compact group such that $G_{0}$ is nilpotent and each compact subset of $G / G_{0}$ generates a compact subgroup of $G / G_{0}$. Then

(1) $\operatorname{RR}\left(C^{*}(G)\right) \leq \max \left\{1, \operatorname{rank}\left(G_{0} /\left[G_{0}, G_{0}\right]\right)\right\}$

(2) $\operatorname{sr}\left(C^{*}(G)\right) \leq \max \left\{2,1+\left\lfloor\frac{1}{2} \operatorname{rank}\left(G_{0} /\left[G_{0}, G_{0}\right]\right)\right\rfloor\right\}$.

Proof. Let $\mathscr{H}$ denote the collection of all compactly generated open subgroups $H$ of $G$. Then $G=\bigcup_{H \in \mathscr{H}} H$ and, for each $H \in \mathscr{H}, H_{0}=G_{0}$ and $H / G_{0}$ is compact. Since $C^{*}(G)$ is the inductive limit of the $C^{*}$-subalgebras $C^{*}(H), \quad \operatorname{RR}\left(C^{*}(G)\right) \leq \sup _{H \in \mathscr{H}} \operatorname{RR}\left(C^{*}(H)\right)$ and $\operatorname{sr}\left(C^{*}(G)\right) \leq$ $\sup _{H \in \mathscr{H}} \operatorname{sr}\left(C^{*}(H)\right)$ (see [14, Lemma 4.1] and [21, Theorem 5.1]). Statements (1) and (2) now follow by applying Lemma 3.2 to each $H \in \mathscr{H}$. 
In the next result, we will use the fact that if $G$ is an almost connected, nilpotent, locally compact group and $G_{0}$ is the connected component of the identity element then $\operatorname{rank}(G /[G, G])=\operatorname{rank}\left(G_{0} /\left[G_{0}, G_{0}\right]\right)[3$, Lemma 1.4] and furthermore $\operatorname{rank}(G /[G, G]) \geq 2$ if $G_{0}$ is not abelian [3, p. 95]. We will also use the fact that if $G$ is an abelian locally compact group then $\operatorname{RR}\left(C^{*}(G)\right)=\operatorname{dim} \hat{G}=\operatorname{rank}(G)$ and $\operatorname{sr}\left(C^{*}(G)\right)=1+\left\lfloor\frac{1}{2} \operatorname{rank}(G)\right\rfloor$ (see, for example, the discussion in [2, p. 2170]).

THeORem 3.4. Let $G$ be an almost connected, nilpotent, locally compact group and let $G_{0}$ be the connected component of the identity element. Then

$$
\begin{aligned}
\operatorname{RR}\left(C^{*}(G)\right) & =\operatorname{rank}(G /[G, G])=\operatorname{rank}\left(G_{0} /\left[G_{0}, G_{0}\right]\right) \\
& =\operatorname{RR}\left(C^{*}\left(G_{0}\right)\right)<\infty \\
\operatorname{sr}\left(C^{*}(G)\right) & =1+\left\lfloor\frac{1}{2} \operatorname{rank}(G /[G, G])\right\rfloor \\
& =1+\left\lfloor\frac{1}{2} \operatorname{rank}\left(G_{0} /\left[G_{0}, G_{0}\right]\right)\right\rfloor=\operatorname{sr}\left(C^{*}\left(G_{0}\right)\right)<\infty
\end{aligned}
$$

Proof. Since $G$ is almost connected, it is compactly generated. So $G /[G, G]$ is a compactly generated abelian group and therefore has finite rank.

(1) Suppose firstly that $\operatorname{rank}\left(G_{0} /\left[G_{0}, G_{0}\right]\right) \geq 1$. Then it follows from Lemma 3.2 that

$$
\begin{aligned}
\operatorname{RR}\left(C^{*}(G)\right) & \leq \operatorname{rank}\left(G_{0} /\left[G_{0}, G_{0}\right]\right)=\operatorname{rank}(G /[G, G]) \\
& =\operatorname{RR}\left(C^{*}(G /[G, G])\right) \leq \operatorname{RR}\left(C^{*}(G)\right),
\end{aligned}
$$

where the final inequality follows from the fact that $C^{*}(G /[G, G])$ is a quotient of $C^{*}(G)$. This establishes the first two equalities of (1), and the final equality follows from replacing $G$ by $G_{0}$.

Now suppose that $\operatorname{rank}\left(G_{0} /\left[G_{0}, G_{0}\right]\right)=0(<2)$. Then $G_{0}$ is abelian and hence, since the rank is zero, it is compact. Since $G / G_{0}$ is compact, we obtain that $G$ is compact and hence that $\operatorname{RR}\left(C^{*}(G)\right)=0=\operatorname{RR}\left(C^{*}\left(G_{0}\right)\right)$, as required.

(2) Suppose firstly that $\operatorname{rank}\left(G_{0} /\left[G_{0}, G_{0}\right]\right) \geq 2$. Then it follows from Lemma 3.2 that

$$
\begin{aligned}
\operatorname{sr}\left(C^{*}(G)\right) & \leq 1+\left\lfloor\frac{1}{2} \operatorname{rank}\left(G_{0} /\left[G_{0}, G_{0}\right]\right)\right\rfloor=1+\left\lfloor\frac{1}{2} \operatorname{rank}(G /[G, G])\right\rfloor \\
& =\operatorname{sr}\left(C^{*}(G /[G, G])\right) \leq \operatorname{sr}\left(C^{*}(G)\right) .
\end{aligned}
$$


This establishes the first two equalities of (2), and the final equality follows from replacing $G$ by $G_{0}$.

Now suppose that $\operatorname{rank}\left(G_{0} /\left[G_{0}, G_{0}\right]\right) \leq 1(<2)$. Then $G_{0}$ is abelian and so

$$
\operatorname{sr}\left(C^{*}\left(G_{0}\right)\right)=1+\left\lfloor\frac{1}{2} \operatorname{rank}\left(G_{0}\right)\right\rfloor=1+\left\lfloor\frac{1}{2} \operatorname{rank}(G /[G, G])\right\rfloor=1 .
$$

So it remains to show that $\operatorname{sr}\left(C^{*}(G)\right)=1$. Let $K$ be a compact normal subgroup of $G$ such that $G / K$ is a Lie group. Then, as in the proof of Lemma 3.2, it suffices to show that $\operatorname{sr}\left(C^{*}(G / K)\right)=1$. As observed in the proof of Lemma 3.2, $G / G_{0} K$ is finite and so $G_{0} K / K$ is an abelian normal subgroup of $G / K$ with finite index. Thus $G / K$ is a Moore group [17, Theorem 1] and furthermore $G_{0} K / K$ is contained in $(G / K)_{F}$ with necessarily finite index. It then follows from [2, Theorem 3.4 and Lemma 2.8] that

$$
\begin{aligned}
1 \leq \operatorname{sr}\left(C^{*}(G / K)\right) & \leq 1+\left\lfloor\frac{1}{2} \operatorname{rank}\left((G / K)_{F}\right)\right\rfloor \\
& =1+\left\lfloor\frac{1}{2} \operatorname{rank}\left(G_{0} K / K\right)\right\rfloor \leq 1+\left\lfloor\frac{1}{2} \operatorname{rank}\left(G_{0}\right)\right\rfloor=1,
\end{aligned}
$$

where the third inequality holds because $G_{0} K / K$ is a quotient of the abelian group $G_{0}$ (see, for example, [3, p. 91]).

The following corollary concerns the 'threadlike' nilpotent Lie groups $G_{N}$ $(N \geq 3$ ), which have been studied by several authors (see [1] and the references cited therein). The group $G_{3}$ is the continuous Heisenberg group. The result for the stable rank is already known as a consequence of [28], but the value of the real rank appears to be new since the rank of $G_{N} /\left[G_{N}, G_{N}\right]$ is even (cf. [3, Corollary 1.6]).

Corollary 3.5. Let $G_{N}$ be a threadlike nilpotent Lie group $(N \geq 3)$. Then $\operatorname{RR}\left(C^{*}\left(G_{N}\right)\right)=2$ and $\operatorname{sr}\left(C^{*}\left(G_{N}\right)\right)=2$.

Proof. Since $G_{N} /\left[G_{N}, G_{N}\right]=\mathrm{R}^{2}$, the result follows from Theorem 3.4.

In view of Lemma 3.2, the question arises as to whether parts (1) and (2) of Theorem 3.4 remain true if only $G_{0}$ is assumed to be nilpotent. We show below that, even when $G_{0}$ is abelian, in both (1) and (2) no two of the first three numbers need be equal.

Example 3.6. Let $G=\mathrm{R}^{n} \rtimes \mathrm{Z}_{2}$ where $n \geq 1$ and $-1 \in \mathrm{Z}_{2}$ acts on $\mathrm{R}^{n}$ by $x \rightarrow-x$. Then the irreducible representations of $G$ are either $1-$ or 2dimensional and $\mathrm{R}^{n}=G_{0}=[G, G]=G_{F}$. In particular, $\operatorname{rank}(G /[G, G])=$ 
$\operatorname{rank}\left(\mathrm{Z}_{2}\right)=0$ and $\left[G: G_{F}\right]=2$. In the following, we apply the results of [2] on the real rank and stable rank of $C^{*}$-algebras of Moore groups.

Let $n=4$. Then $\operatorname{rank}\left(G_{F}\right)=4$ and it follows from [2, Theorem 3.4] that

$$
2 \leq \operatorname{RR}\left(C^{*}(G)\right) \leq \operatorname{RR}\left(C^{*}\left(G_{F}\right)\right)=4
$$

and

$$
2 \leq \operatorname{sr}\left(C^{*}(G)\right) \leq \operatorname{sr}\left(C^{*}\left(G_{F}\right)\right)=3 .
$$

On the other hand, since $\operatorname{rank}\left(G_{F}\right)=4$ and $G_{F}^{c}=\{0\}$, it follows from [2, Theorem 4.3] that $\operatorname{RR}\left(C^{*}(G)\right) \neq \operatorname{RR}\left(C^{*}\left(G_{F}\right)\right.$ ) (and in fact the proof of [2, Theorem 4.3] shows that $\operatorname{RR}\left(C^{*}(G)\right)$ is 2 rather than 3) and it follows from [2, Theorem 4.4] that $\operatorname{sr}\left(C^{*}(G)\right) \neq \operatorname{sr}\left(C^{*}\left(G_{F}\right)\right)$. Thus the three numbers $\operatorname{RR}\left(C^{*}(G)\right), \operatorname{RR}\left(C^{*}\left(G_{0}\right)\right)$ and $\operatorname{rank}(G /[G, G])$ are distinct and so are the three numbers $\operatorname{sr}\left(C^{*}(G)\right), \operatorname{sr}\left(C^{*}\left(G_{0}\right)\right)$ and $1+\left\lfloor\frac{1}{2} \operatorname{rank}(G /[G, G])\right\rfloor$.

We note in passing that, for the real rank alone, it suffices to take $n=2$. For then similar arguments show that $\operatorname{RR}\left(C^{*}(G)\right)=1$ and $\operatorname{RR}\left(C^{*}\left(G_{0}\right)\right)=2$.

\section{REFERENCES}

1. Archbold, R. J., Kaniuth, E., Ludwig, J., Schlichting, G., and Somerset, D. W. B., Strength of convergence in duals of $C^{*}$-algebras and nilpotent Lie groups, Adv. Math. 158 (2001), 26-65.

2. Archbold, R. J., and Kaniuth, E., Stable rank and real rank for some classes of group $C^{*}$ algebras, Trans. Amer. Math. Soc. 357 (2005), 2165-2186.

3. Archbold, R. J., and Kaniuth, E., On the stable rank and real rank of group $C^{*}$-algebras of nilpotent locally compact groups, Math. Scand. 97 (2005), 89-103.

4. Brown, L. G., On higher real and stable ranks for CCR $C^{*}$-algebras, arXiv:0708.3072.

5. Brown, L. G., and Pedersen, G. K., $C^{*}$-algebras of real rank zero, J. Funct. Anal. 99 (1991), 131-149.

6. Brown, L. G., and Pedersen, G. K., Limits and $C^{*}$-algebras of low rank or dimension, J. Operator Theory 61 (2009), 381-417.

7. Corwin, L., and Greenleaf, F. P., Representations of Nilpotent Lie Groups and their Applications. Part 1: Basic Theory and Examples, Cambridge Studies Adv. Math. 18, Cambridge Univ. Press, Cambridge 1990.

8. Dixmier, J., Les $C^{*}$-algèbres et leurs représentations, Cahiers Sci. XXIX, Gauthier-Villars, Paris 1964.

9. Dixmier, J., Sur le dual d'un groupe de Lie nilpotent, Bull. Sci. Math. (2) 90 (1966), 113-118.

10. Dykema, K. J., Haagerup, U. and Rørdam, M., The stable rank of some free product $C^{*}$ algebras, Duke Math. J. 90 (1997), 95-121.

11. Dykema, K. J., and de la Harpe, P., Some groups whose reduced $C^{*}$-algebras have stable rank one, J. Math. Pures Appl. (9) 78 (1999), 591-608.

12. Grosser, S., and Moskowitz, M., Compactness conditions in topological groups, J. Reine Angew. Math. 246 (1971), 1-40.

13. Hewitt, E., and Ross, K. A., Abstract Harmonic Analysis I, Grundl. math. Wiss. 115, Springer, Berlin 1963. 
14. Kaniuth, E., Group $C^{*}$-algebras of real rank zero or one, Proc. Amer. Math. Soc. 119 (1993), 1347-1354.

15. Kucerovsky, D., and Ng, P. W., Infinite stable rank for a continuous field algebra with fibres $\mathscr{K}$, J. Operator Theory 54 (2005), 377-386.

16. Montgomery, D., and Zippin, L., Topological Transformation Groups, Interscience, New York 1955.

17. Moore, C. C., Groups with finite dimensional irreducible representations, Trans. Amer. Math. Soc. 166 (1972), 401-410.

18. Nagisa, M., Stable rank of some full group $C^{*}$-algebras of groups obtained by the free product, Internat. J. Math. 8 (1997), 375-382.

19. Nistor, V., Stable range for a certain class of type $I C^{*}$-algebras, J. Operator Theory 17 (1987), 365-373.

20. Pears, A. R., Dimension Theory of General Spaces, Cambridge Univ. Press, Cambridge 1975.

21. Rieffel, M. A., Dimension and stable rank in the $K$-theory of $C^{*}$-algebras, Proc. London Math. Soc. (3) 46 (1983), 301-333.

22. Schulz, E., The stable rank of crossed products of sectional $C^{*}$-algebras by compact Lie groups, Proc. Amer. Math. Soc. 112 (1991), 733-744.

23. Sheu, A.J.-L., A cancellation theorem for modules over the group $C^{*}$-algebras of certain nilpotent Lie groups, Canad. J. Math. 39 (1987), 365-427.

24. Sudo, T., Stable rank of the reduced $C^{*}$-algebras of non-amenable Lie groups of type I, Proc. Amer. Math. Soc. 125 (1997), 3647-3654.

25. Sudo, T., Stable rank of the $C^{*}$-algebras of amenable Lie groups of type I, Math. Scand. 84 (1999), 231-242.

26. Sudo, T., Dimension theory of group $C^{*}$-algebras of connected Lie groups of type I, J. Math. Soc. Japan 52 (2000), 583-590.

27. Sudo, T., Survey on the stable rank of group $C^{*}$-algebras of two-step nilpotent groups, Ryukyu Math. J. 14 (2001), 67-77.

28. Sudo, T., and Takai, H., Stable rank of the $C^{*}$-algebras of nilpotent Lie groups, Internat. J. Math. 6 (1995), 439-446.

29. Sudo, T., and Takai, H., Stable rank of the $C^{*}$-algebras of solvable Lie groups of type I, J. Operator Theory 38 (1997), 67-86.

INSTITUTE OF MATHEMATICS

UNIVERSITY OF ABERDEEN

ABERDEEN AB24 3UE

SCOTLAND

UNITED KINGDOM

E-mail: r.archbold@abdn.ac.uk
INSTITUTE OF MATHEMATICS UNIVERSITY OF PADERBORN 33095 PADERBORN

GERMANY

E-mail: kaniuth@math.uni-paderborn.de 